\title{
Assessment-focused Model for Monitoring Student Attributes
}

\author{
D MacIsaact ${ }^{\dagger+}$, C Diduch ${ }^{\dagger}$, E Hussein ${ }^{\dagger}$ \\ tFaculty of Engineering, ${ }^{+t}$ Center for Enhanced Teaching and Learning, University of New Brunswick \\ hussein@unb.ca
}

\begin{abstract}
Faculty at the University of New Brunswick have worked collaboratively to develop a streamlined monitoring process for graduate attributes intended to be easy to understand, efficient, and comply with intentions laid out by the Canadian Engineering Accreditation Board. The monitoring process is made up of two parts: An assessment-focused model for monitoring student progress, and a course mapping exercise for monitoring learning opportunities. In monitoring student progress, typical student assessments are used as opportunities for students to demonstrate that expectations are being met in the context of attributes. This provides a transparent mechanism for instructors to produce evidence that their students are developing attributes. To date, expectations for six of the twelve attributes have been articulated in a rubric, and four of the attributes have been tracked. Our experience thus far indicates that our monitoring process allows us 1) to uniformly express expectations regarding graduating student attributes across programs, 2) to indentify assessments which provide opportunities for our students to demonstrate the behaviors outlined in our expectations, and 3) to use results of the assessments to easily summarize data about the attributes of our graduating students.
\end{abstract}

Keywords: CEAB attributes, streamlined continuous curriculum improvement program, expectations checklist.

\section{INTRODUCTION}

Since the Canadian Engineering Accreditation Board (CEAB) began requiring engineering programs to assess attributes of engineering students in 2009 [1], most universities across Canada have started to work on how best to do this at their institutions. A complementary requirement, also explicitly articulated by the $\mathrm{CEAB}$, is to ensure that our programs continually improve. More specifically, the CEAB requires that accredited programs have "processes in place that demonstrate that program outcomes are being assessed in the context of the graduate attributes, and that the results are applied to the further development of the program" [2].
Recently at the University of New Brunswick (UNB), representatives from each of our undergraduate engineering programs have been working collaboratively to develop a framework within which we can meet these goals. We have adopted the term Continuous Curriculum Improvement Process (CCIP) from McCahan et al [3] to describe our framework, which includes 1) a monitoring process used to track student attributes and learning opportunities and 2) a reaction processes used to change our programs to better ensure that graduating students acquire critical attributes as delineated by the CEAB. For convenience, the twelve attributes are listed below [2]:

- A knowledge base for engineering

- Problem Analysis

- Investigation

- Design

- Use of Engineering Tools

- Individual and Team Work

- Communication Skills

- Professionalism

- Impact of Engineering on Society and Environment

- Ethics and Equity

- Economics and Project Management

- Life-long Learning

Figure 1 depicts an overview of our framework including both the monitoring and reaction processes. In order to ensure that we have sufficient information to identify effective reaction strategies from the information acquired during the monitoring process, our CCIP has been designed to provide information about student abilities not only at the end of their program (attributes of graduating students), but also at some reasonable input stages (attributes of continuing students, i.e. year 1, 2 and/or 3). Information about learning opportunities between these stages is also important, so the CCIP also includes monitoring of our curriculum. These are typical practices to be included within any adopted CCIP [1], [3],[4] and the information gathered collectively from these monitoring process can be fed into a reaction process to drive positive change. 


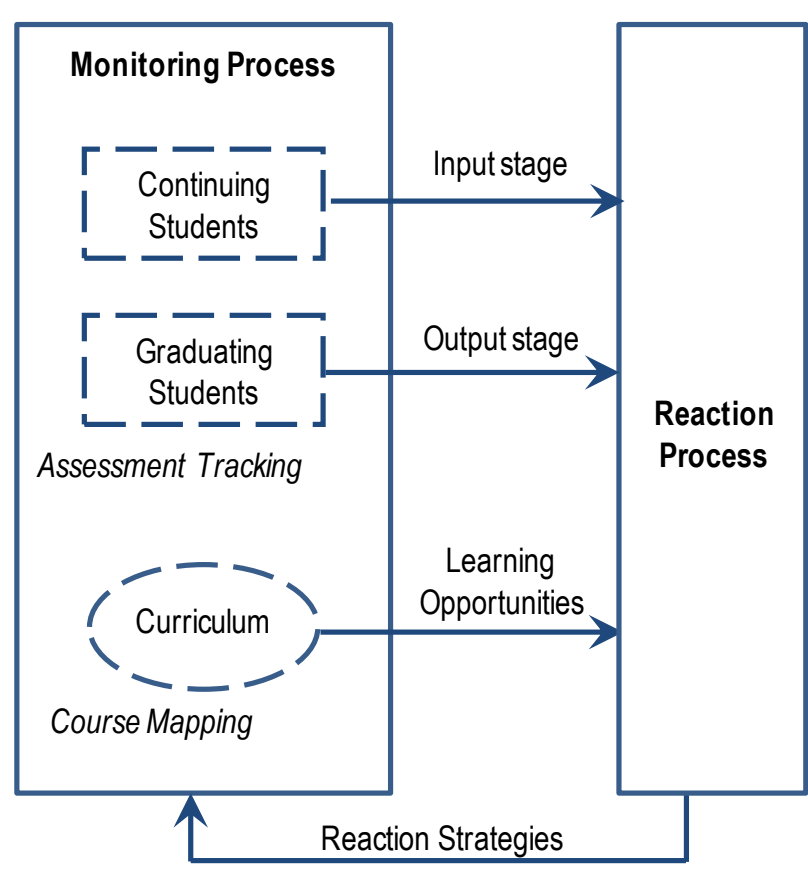

Fig. 1. Framework for the UNB continuous curriculum improvement process

There is nothing particularly inventive about our framework; however, we have been fortunate at UNB to have healthy involvement in its development from representatives across all of our programs. This has helped us to ensure that our CCIP considers aspects of feasibility and impact at the faculty level, the program level, and the instructor level. Our intentions have been to develop a CCIP that complies with the mandates of the $\mathrm{CEAB}$ and helps us to continue to deliver quality programs, but also to develop a CCIP that is:

- easy to understand for a) instructors who have to implement it, and b) visitors who will be reviewing the results

- efficient - requiring limited time commitment while generating measurable impact

- flexible - to ensure a single framework which suits the needs and standards for all our programs

To meet these goals, we have designed our framework to be one that is self-evolving. The feedback loop depicted in Fig. 1 (monitoring-reaction-monitoring) is meant to imply that analysis of data collected through monitoring should be used not only to drive changes in our curriculum that positively influence our students, but also to drive changes to improve our monitoring processes. This approach gives us leeway to build incrementally to a set of fully sustainable, evidence-informed processes and to start by examining, in a way that is easy for our instructors to understand, our current practices. As our instructors grow more knowledgeable through participation in our CCIP, so too will the complexity of how we monitor and react. However, to ensure efficiency promote compliance, we have purposefully started simply, and will add complexity only as needed. Based on results of our preliminary use, our collaborative and self-evolving approach is paying off. Aspects of CCIP ready for trial have been promoted for use and managed by the faculty, supported through varying approaches to coordination at the program level, and agreeably adopted by instructors.

Our framework is a work in progress and the reaction process has yet to be specified. We have developed a preliminary means by which we monitor our curriculum via a course mapping exercise. The contents of all core courses are mapped against a set of expectations delineated faculty-wide for each of the twelve attributes. Currently, these course mappings form the basis of our articulation of learning opportunities in each of our programs. While we recognize the importance of expanding and intensifying this aspect of the process, for now our focus has been directed at developing the monitoring processes involved with tracking student attributes. We have based our efforts around an assessment-focused model similar to that promoted by the Engineering Graduate Attribute Project (EGAD) [5], and the purpose of this paper is to detail the processes based on this model.

\section{MONITORING STUDENT ATTRIBUTES}

Figure 2 depicts the artifacts generated and used in our framework to track student progress in the context of the CEAB attributes. First, a set of faculty-wide expectations for behaviors is articulated for each attribute. The expectations are expressed in a rubric (one for each attribute), which delineates behaviors at four levels:

- Level 1: describes behaviors that are below what is expected for graduating students

- Level 2: describes behaviors that are at the threshold of what is expected for graduating students

- Level 3: describes behaviors that are at the target for what is expected for graduating students

- Level 4: describes behaviors that exceed what is expected for graduating students

Articulating expectations for behaviors at each of these levels is an important aspect of the monitoring process. First, since we are collecting data for continuing students as well as graduating students, behaviors that are below threshold are also under consideration. Second, boundary conditions such as those expressed in the threshold and exceeds levels, are useful in establishing mutual understanding about the behaviors in the target level. 


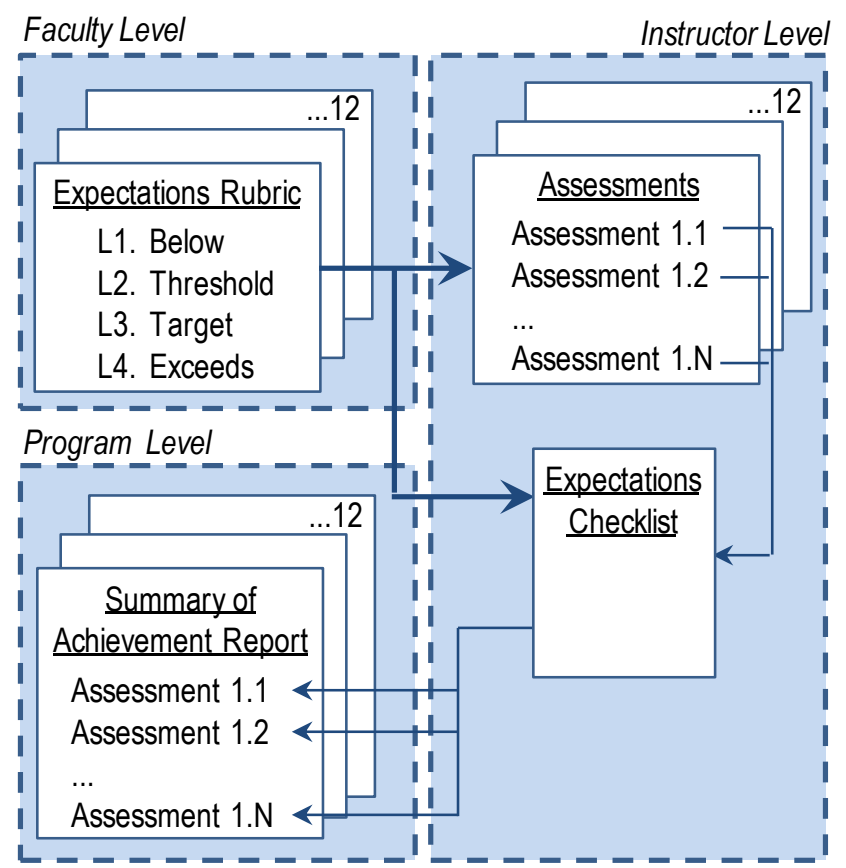

Fig. 2. Assessment-focused model used at UNB to monitor student attributes

With well-formed expectations in hand, instructors can identify assessment tools (labs, assignments, tests, reports, class discussions, etc) that they currently implement in their courses (or elsewhere) which can be used to provide evidence of expected behaviors. Not all assessment tools identified need to be tracked. Many articulated behaviors are likely to be assessed in multiple places within a program and it is not our intention to track every instance. Instead, particular assessment tools may be selected for tracking. Typically, more than one will need to be selected to cover all of the behaviors listed in a rubric, to collect data on both continuing and graduating students, and to cover most students in a program, multiple times. To be most efficient, we have recommended that our programs choose assessments which 1) cover the broadest range of the expectations listed in the rubric, and 2) cover the largest sample of students.

Those instructors responsible for the selected assessment tools are responsible for tracking student performance on the assessments, as it relates to expected behaviors. To help instructors with this, they are provided with an expectations checklist. This checklist includes a list of all of the expected behaviors for a given attribute classified by level (reproduced from the rubric), and instructors can simply check-off which behaviors are addressed in their assessment tool. As an example, Table 1 delineates the contents of our expectations checklist for the communications attribute. Since an assessment may be selected to provide evidence for more than one attribute, a checklist is provided for each relevant attribute (usually between 1 and 3 ). The checklist also provides a summary table for instructors to record the number (and percentage) of students who performed at each level based on their assessment.

Data from the expectations checklists are collected for each program and compiled into a summary of achievement report for each attribute. This report includes a table which lists the data collected from each assessment tool that was tracked, and commentary about items in the list and/or collective observations. As an example, Table 2 delineates the contents of a summary of achievement report for the communications attribute and our software engineering program.

All of the artifacts described here will be made available to visitors during a $\mathrm{CEAB}$ review. The expectation rubrics provide an articulation of what behaviors we expect from our students in terms of the CEAB attributes. The summary of achievement reports provide a snapshot of what behaviors our students are demonstrating in the context of their attributes. The checklists, together with samples of graded assessments provide evidence to support what is reported in the snapshot data.

Figure 2 depicts our monitoring process from three distinct perspectives. At the instructor level, student assessments are the focus, which is why we have termed it the assessment-focused model. At the program level, assessment tracking across courses that comprise individual programs are consolidated. While working within the guidelines provided by the faculty, each program works autonomously to organize and coordinate ongoing monitoring and consolidation. Our faculty guidelines specify a three year cycle for monitoring, roughly 4 attributes/year according to the following schedule:

- Year-1: Investigation, Communication Skills, Design, and Use of Engineering Tools

- Year-2: Problem Analysis, Economics and Project Management, Individual and Team Work, Professionalism

- Year-3: Impact of Engineering on Society and Environment, Ethics and Equity, Life-long Learning

Monitoring the knowledge-base for engineering attribute will be handled yearly, through a process yet to be fully specified. At the faculty level, a CCIP committee made up of representatives from each of our programs is responsible for maintaining a common archive for storing and retrieving the standard data consolidated by each program, for providing guidelines and direction to programs to ensure a coordinated implementation of the CCIP, and for setting and updating the expectation rubrics and checklists. 
Table 1: Contents of expectations checklist for communications attribute (levels 1-4 are indicated by L1, L2, L3 and L4) Total \# of students:

\begin{tabular}{|l|l|l|l|l|l|}
\hline $\begin{array}{l}\text { (Level 1) } \\
\begin{array}{l}\text { \# students below } \\
\text { threshold }\end{array}\end{array}$ & $\begin{array}{l}\text { (Level 1) } \\
\text { \# students at } \\
\text { threshold }\end{array}$ & & $\begin{array}{l}\text { (Level 1) } \\
\text { \# students at } \\
\text { target }\end{array}$ & & $\begin{array}{l}\text { (Level 1) } \\
\text { \# students } \\
\text { exceeding target }\end{array}$ \\
\hline
\end{tabular}

Verbal (Speaking, listening, discussing)

(L1) Respond appropriately to clear verbal instructions.

(L1) Ask and answer questions one-on-one and in groups to clarify engineering concepts.

(L1) Explain engineering concepts through one-on-one discussions.

(L1) Verbally describe engineering concepts to a specified audience.

(L1) Respectfully participate in discussion.

(L2) Organize and verbally deliver ( $\square$ (L3) concise) explanations about engineering concepts and projects to lead respectful discussions one-onone and with heterogeneous audiences.

(L3) Deliver clear verbal instructions.

(L3) Recognize and construct credible arguments to verbally participate in/ ( $\square$ (L4) conduct) civil negotiations to establish mutual agreement.

(L4) User verbal eloquence to responsibly persuade negotiators to establish mutual agreement.

Written (textual reading and writing)

(L1) Respond appropriately to clearly written instructions.

$\square$ (L1) Apply the contents of required readings/ ( $\square$ (L2) supplemental readings) to form explanations for engineering concepts ( $\square$ (L2) and to present evidence to support a claim).

(L1) Apply the rules of grammar ( $\square$ (L2) and composition) in written communications.

(L1) Given a set of instructions detailing format, structure, and contents/ ( $\square$ (L2) suggestions regarding contents and length) compose the elements of a written communication, using respectful language and properly citing sources.

(L3) Identify credible written sources.

(L3) Plan and compose ( $\square$ (L4) concisely) written communications of a project for a heterogeneous readership, using respectful language and properly citing sources.

(L4) Use written eloquence to responsibly persuade readers towards a point of view.

Graphical (engineering data and drawings)

(L1) Accurately interpret data represented in graphical elements such as tables, charts, plots and engineering diagrams ( $\square$ (L4) representing multi-disciplinary concepts).

(L1) Given templates for graphical elements / ( $\square$ (L2) a set of instructions and drawing standards), tabulate and plot data for written communications and verbal presentations.

(L1) Draw sketches and diagrams to support verbal and written explanations for engineering concepts.

(L3) Organize data for reporting ( $\square$ (L4) multi-disciplinary concepts) with appropriate use of tables, charts, and plots.

(L3) Use appropriate graphical standards in preparing sketches and engineering drawings.

(L3) Integrate graphic elements appropriately into written communications and verbal presentations.

Virtual (computer authoring tools)

(L1) Use a specified tool and visual templates ( $\square$ (L2) and exploit their features) to compile data into tables, charts.

(L1) Use a specified tool and appropriate graphical standards in preparing engineering drawings.

(L1) Use specified/ ( $\square$ (L3) appropriate) security protocols, posting guidelines, and etiquette when communicating with virtual tools (such as email, wikis, blogs, web sites).

(L2) Use a set of specified tools and exploit their features in preparing engineering drawings to appropriate graphical standards.

(L2) Use a specified tool to integrate graphical and textual components within verbal and written communications.

$\square$ (L3) Choose appropriate tools from a set of tools provided/ ( $\square$ (L4) conduct an open technology assessment to choose a set of tools), and exploit their features to compile data in to tables, charts and plots, to prepare engineering drawing using appropriate graphical standards and to integrate graphical and textual components within verbal and written communications.

Some of our departments have set up program assessment committees to oversee the monitoring process for their programs. Others have assigned each of their faculty members to oversee the monitoring process of one or more attributes. Still others are relying on their Chairs to ensure that monitoring takes place. Regardless of how it is coordinated, each program must ensure that a set of assessment tools is identified for monitoring, and that the instructors responsible for those assessments provide the tracking data necessary to complete the summary of achievement reports, along with samples of the assessments used to collect the data. 
Table 2: Contents of summary of achievement report for communications attribute in software engineering program

\begin{tabular}{|c|c|c|c|c|c|c|}
\hline Group & Indicator & Sample Size & \%Below* & \%Threshold* & $\%$ Target $^{*}$ & \%Exceeding* \\
\hline Graduating & $\begin{array}{c}\text { SWE4103 Report }{ }^{1} \\
\text { SWE4103 Presentation² } \\
\text { SWE4103 Questions }^{3}\end{array}$ & $\begin{array}{l}6 \\
6 \\
6\end{array}$ & $\begin{array}{c}(66 \%) 4 \\
0 \\
(66 \%) 4\end{array}$ & $\begin{array}{c}0 \\
(16 \%) 1 \\
0\end{array}$ & $\begin{array}{c}0 \\
(66 \%) 4 \\
0\end{array}$ & $\begin{array}{l}(33 \%) 2 \\
(16 \%) 1 \\
(33 \%) 2\end{array}$ \\
\hline Year 4+ & $\begin{array}{c}\text { SWE4103 Report1 } \\
\text { SWE4103 Presentation² } \\
\text { SWE4103 Questions }{ }^{3}\end{array}$ & $\begin{array}{l}6 \\
6 \\
6 \\
\end{array}$ & $\begin{array}{l}0 \\
0 \\
0\end{array}$ & $\begin{array}{l}(33 \%) 2 \\
(33 \%) 2 \\
(33 \%) 2\end{array}$ & $\begin{array}{l}(33 \%) 2 \\
(50 \%) 3 \\
(33 \%) 2 \\
\end{array}$ & $\begin{array}{l}(33 \%) 2 \\
(16 \%) 1 \\
(33 \%) 2\end{array}$ \\
\hline \multicolumn{7}{|l|}{ Year 3} \\
\hline \multicolumn{7}{|l|}{ Year 2} \\
\hline Year 1 & ENGG 1013 Report on an Engineer ${ }^{4}$ & 15 & $(13 \%) 2$ & $(87 \%) 13$ & $\mathrm{~N} / \mathrm{A}$ & $\mathrm{N} / \mathrm{A}$ \\
\hline \multicolumn{7}{|c|}{$\begin{array}{l}\text { 1SWE4103 Report: Evaluated Written and Graphical and Virtual aspects of communications attribute (limited engineering diagrams); weaknesses in } \\
\text { following written and verbal instructions was noted (see checklist for details) }\end{array}$} \\
\hline \multicolumn{7}{|c|}{$\begin{array}{l}\text { 2SWE4103 Presentation: Evaluated Verbal, Graphical and Virtual aspects of communications attribute (limited engineering diagrams) } \\
\text { weaknesses in following written and verbal instructions was noted (see checklist for details) }\end{array}$} \\
\hline \multicolumn{7}{|c|}{ 3SWE4103 Questions: Evaluated providing written instructions (see checklist for details) } \\
\hline \multicolumn{7}{|c|}{${ }^{4}$ ENGG 1013 Report on an Engineer: Evaluated Written and Virtual aspects of communications attribute (see checklist for details) } \\
\hline \multicolumn{7}{|c|}{$\begin{array}{l}\text { Most students ( } 87 \%) \text { come into our program at the threshold level of writing skills. There is a distinct difference in performance between Verbal } \\
\text { aspects of communication and Written aspects in the upper years. Conservatively } 100 \% \text { of our graduating students are at the threshold or better for } \\
\text { Verbal aspects of this attribute and } 33 \% \text { of our graduating students are at the threshold or better for Written aspects of this attribute. While no } \\
\text { students fall below our expectations for verbal, } 66 \% \text { of our students fall below expectations for written. While the assessment of year } 1 \text { and } \\
\text { graduating students is based on different samples, it is worth noting that the current data shows a decline in written skills. This could be a difference } \\
\text { in sample populations, or a difference in assessment process, or assessors and should be further observed. This sample represents } 100 \% \text { of our } \\
\text { total population of graduating students. It is worth noting that students preparing to graduate (one year to completion) all performed at the threshold } \\
\text { level or better. Also of note is that we are not assessing ability to draw engineering diagrams, and that a weakness in following verbal instructions } \\
\text { was reported. }\end{array}$} \\
\hline
\end{tabular}

\section{PRELIMINARY FINDINGS}

With the summary of achievement reports compiled, our hope is that programs will be able to analyse the data to:

- Identify attributes which are not currently being assessed so that new assessments (and subsequent learning opportunities) may be introduced when necessary

- Identify attributes that are being poorly assessed (indicated by contradictory data and/or through assessment review) so that improved assessments may be introduced when necessary

- Identify attributes that are being assessed well but are not being met so that better learning opportunities may be introduced when necessary

- Identify attributes for which our expectations are being surpassed so that we use these as opportunities to increase our expectations or streamline our curriculum.

While we do not have our reaction processes specified, we are currently completing year-1 of our first cycle of monitoring with our new process and are just now analyzing the compiled results.

We are encouraged to report that our programs were able to solicit instructors willing to track student progress with relevant assessments for each of the four attributes monitored this year. We encountered initial push-back as instructors found it challenging to map the contents of our rubrics to their assessment tools. This lead to the introduction of expectation checklists, which eased the process significantly, since instructors could indicate more specifically which behaviors were being tracked and at what level.

Many instructors indicated that it was still difficult to assign each student to a level, since most students demonstrated behaviors at more than one level in a given assessment. This difficulty was overcome in many cases by breaking the tracking process into components (based on rubric columns), since often students demonstrate behaviors at one level for a particular aspect of an attribute (for instance, L1 for written communication skills), and another level for a different aspect (for instance, L3 for verbal communication skills). Table 2 provides an example of this, where the SWE4103 seminar was split into report and presentation components, and a tracking entry for each was recorded. Instructors also 
indicated that the freeform commentary was useful, and essential, for cases which could not be easily itemized.

We are also encouraged to report that most of our participating instructors found it reasonably easy to interpret the articulated expectations, and to find aspects of their assessment tools that corresponded to them. Our preliminary review of the assessment tools submitted indicate that they matched well with what we intended for assessment as expressed in the expectation rubrics. Furthermore, we have anecdotal reports that instructors have learned from their experiences with the CCIP this year and plan to modify their assessment tools next year to 1) comply more thoroughly with our program expectations, and 2) become more transparent in the way their assessment tools map to their assessment of levels of achievement in their students.

Finally, though many of our programs have yet to fully analyse the compiled results, feedback has indicated that we are able to make observations from the data that will be useful in the reaction process. For instance, as delineated in the summary of Table 2, data indicate that graduating students in our software engineering program are meeting our verbal communication expectations, but not our written ones. As a result of this process, we are now looking at ways to increase learning opportunities for our software engineering students in this area.

While we are encouraged by our initial findings, we also recognize some important limitations. In this first cycle, our intention has been to focus on assessment tools for direct evidence of learning which are already being implemented in our courses. While we were successful this year in finding tools that met our needs, this focus will eventually have to expand for varied reasons. First, a richer data set would likely be obtained by supplementing these tools with some tools which provide indirect evidence of learning such as that which may be collected from student focus groups and alumni surveys. Moreover, it is likely that some attributes will require the use of assessment tools for indirect evidence of learning. Second, the assessment tools currently in focus were not specifically designed as opportunities to demonstrate expected behaviors in the context of graduating attributes. As some of our instructors have indicated, this can make it challenging to map demonstrated achievement into an expectation level. Nevertheless, we maintain that this is a critical first step in the process at our institution. Our approach is allowing instructors to explore the concepts of data-informed curriculum improvement from a perspective with which they are familiar. Our experiences thus far indicate that this may indeed be instrumental in achieving compliance of a sustainable, comprehensive and effective CCIP.

\section{CONCLUSIONS}

UNB has been working hard to implement a CCIP that complies with the mandates of the CEAB and helps us to continue to deliver quality programs. Our approach has been to develop a framework which will allow us to start in simple terms and grow in complexity as needed. To this end, we have articulated faculty-wide expectations in terms of behaviors which demonstrate the graduate attributes, and offered our faculty opportunities to explore how to best assess these behaviors in ways that are familiar to them. All of our programs have embraced our framework thus far, and after the completion of year-1 in our first three year cycle, we have data about four attributes which can be used to inform curriculum and our evolving framework, and instructors who are eager to make changes in the way they teach and assess, to better meet the needs of our students. While we are at the start of an ongoing process, we are encouraged by our success thus far.

\section{Acknowledgements}

The work presented here represents the collective effort of the UNB engineering CCIP working group. Besides the authors, members of this group include, $\mathrm{B}$ Bramble, K Butler, W Cooke, P Dare, A Gerber, M Kaye, B Lowry, and B Wilson.

\section{References}

[1] Brian Frank, Susan McCahan, K Christopher Watts, Susan Fostaty-Young, Peter Ostafichuck, Perter Wolf, Nasser Saleh, "Engineering graduate attribute (EGAD) project," in Proceedings of the $2^{\text {nd }}$ Annual Canadian Engineering Education Association (CEEA) Conference, St John's NF, 2011.

[2] Canadian Accreditation Board, Accreditation Criteria and Procedures, 2011. p14. Available as of May 08, 2012 from http://www.engineerscanada.ca/files/w Accreditation Crite ria Procedures 2011.pdf.

[3] Susan McCahan, Lisa Romkey, Grant Allen, "Development of the graduate attribute quality assurance process at the University of Toronto," in Proceedings of the $2^{\text {nd }}$ Annual Canadian Engineering Education Association (CEEA) Conference, St John's NF, 2011.

[4] David Strong, Sue Fostaty Young, "Consideration of the design attribute in the 2008 CEAB accreditation procedures," in Proceedings of the Canadian Engineering Education Association (CEEA) Conference, Kingston ON, 2010 .

[5] Canadian Engineering Accreditation Board, Questionnaire for Evaluation of an Engineering Program, Available as of May $12^{\text {th }} 2012$ from http://egad.engineering.queensu.ca/. 